\title{
Investigation of the Neupert effect in solar flares
}

\section{Statistical properties and the evaporation model}

\author{
A. Veronig ${ }^{1}$, B. Vršnak ${ }^{2}$, B. R. Dennis ${ }^{3}$, M. Temmer ${ }^{1}$, A. Hanslmeier ${ }^{1}$, and J. Magdalenić 2 \\ 1 Institute for Geophysics, Astrophysics and Meteorology, University of Graz, Universitätsplatz 5, 8010 Graz, Austria \\ 2 Hvar Observatory, Faculty of Geodesy, University of Zagreb, Kačićeva 26, HR-10000 Zagreb, Croatia \\ 3 NASA Goddard Space Flight Center, Greenbelt, MD 20771, USA
}

Received 11 April 2002 / Accepted 6 June 2002

\begin{abstract}
Based on a sample of 1114 flares observed simultaneously in hard X-rays (HXR) by the BATSE instrument and in soft X-rays (SXR) by GOES, we studied several aspects of the Neupert effect and its interpretation in the frame of the electronbeam-driven evaporation model. In particular, we investigated the time differences $(\Delta t)$ between the maximum of the SXR emission and the end of the HXR emission, which are expected to occur at almost the same time. Furthermore, we performed a detailed analysis of the SXR peak flux - HXR fluence relationship for the complete set of events, as well as separately for subsets of events which are likely compatible/incompatible with the timing expectations of the Neupert effect. The distribution of the time differences reveals a pronounced peak at $\Delta t=0$. About half of the events show a timing behavior which can be considered to be consistent with the expectations from the Neupert effect. For these events, a high correlation between the SXR peak flux and the HXR fluence is obtained, indicative of electron-beam-driven evaporation. However, there is also a significant fraction of flares (about one fourth), which show strong deviations from $\Delta t=0$, with a prolonged increase of the SXR emission distinctly beyond the end of the HXR emission. These results suggest that electron-beam-driven evaporation plays an important role in solar flares. Yet, in a significant fraction of events, there is also clear evidence for the presence of an additional energy transport mechanism other than nonthermal electron beams, where the relative contribution is found to vary with the flare importance.
\end{abstract}

Key words. Sun: flares - Sun: X-rays, gamma rays - Sun: corona - Sun: chromosphere - methods: statistical

\section{Introduction}

Observations of solar flares in X-rays and microwaves frequently show that the shape of the rising part of the soft $\mathrm{X}$-ray light curve closely resembles the time integral of the microwave or hard X-ray light curve. This led to the idea that there is a causal relationship between the nonthermal (microwave and hard X-ray) and thermal (soft X-ray) emission of a flare (Neupert 1968; Dennis \& Zarro 1993), which has become known as the Neupert effect.

It has been shown that this effect can be reproduced by a model, in which the flare energy is released primarily in the form of nonthermal electrons (e.g., Brown 1971; Li et al. 1993). According to the so-called thick-target model, the hard X-ray (HXR) emission is electron-ion bremsstrahlung produced by electron beams encountering the dense layers of the lower corona, transition region, and chromosphere. The model assumes that only a small fraction of the energy of the nonthermal electrons is lost through radiation (for a discussion see McDonald et al. 1999). Most of the energy is transferred to

Send offprint requests to: A. Veronig, e-mail: asv@igam.uni-graz.at heating of the ambient thick-target plasma via Coulomb collisions between the beam and the ambient electrons. Due to the rapid deposition of energy by the accelerated electrons, the energy cannot be radiated away at a sufficiently high rate and strong pressure gradients develop. The pre-flare hydrostatic equilibrium is lost and the heated plasma explosively expands up into the corona in a process known as chromospheric evaporation (e.g., Antonucci et al. 1984; Fisher et al. 1985; see also the review by Antonucci et al. 1999, and references therein). The hot dense plasma that has been convected into the corona gives rise to enhanced soft X-ray (SXR) emission via thermal bremsstrahlung.

Under such circumstances, the hard X-ray emission is directly related to the electron beam flux. On the other hand, the soft X-ray emission should be directly linked to the energy deposited by the same electrons up to a given time, i.e. to the time integral of the electron beam flux, and we can expect to see the Neupert effect. Thus, if the Neupert effect is observed, this can be considered as evidence of electron-beamdriven chromospheric evaporation (see McTiernan et al. 1999). In recent years, the Neupert effect has also been observed on stars, which suggests the existence of the chromospheric 
evaporation process also in stellar flares (Hawley et al. 1995; Güdel et al. 1996).

In the present study we utilize statistical properties of solar flares observed simultaneously in SXR and HXR emission to test several expectations from the Neupert effect. The main predictions are: (1) The maximum of the SXR emission and the end of the HXR emission should occur at the same time. (2) There should be a high correlation between the HXR fluence, i.e. the HXR flux integrated over the event duration, and the SXR peak flux. The correlation of the HXR fluence and the SXR peak flux and its relation to the involved nonthermal and thermal energies provide the fundamental link between the Neupert effect and the electron-beam-driven chromospheric evaporation model, which will be discussed in Sect. 3. The present analysis can be considered as a complementary approach to studies of the Neupert effect which use the actual SXR and HXR light curves (see, e.g., Dennis et al. 1992; Dennis \& Zarro 1993; McTiernan et al. 1999). By accessing only statistical flare quantities, such as HXR end time, SXR maximum time, HXR fluence and SXR peak flux, we neglect part of the information contained in the light curves. However, such a statistical approach has the advantage that it can be applied to a large data set, i.e. it is not restricted to a selected sample of events, which intrinsically favors the analysis of large flares. Moreover, it enables us to define and investigate subsets of events, still representing statistically meaningful data sets.

The paper is structured in the following way. Section 2 contains a description of the soft X-ray and hard X-ray data used in the analysis together with the method of finding corresponding SXR/HXR events. In Sect. 3, we discuss, in the frame of the electron-beam-driven chromospheric evaporation model, the relationship between SXR and HXR emissions, and the associated thermal/nonthermal energies. In this respect, it is essential to clarify the question if and in which formulation the Neupert effect is valid for the bulk of solar flares. In Sect. 4, our results are presented, comprising an investigation of the relative timing of the SXR and HXR emission as well as a detailed study of the HXR fluence - SXR peak flux relationship. The results are interpreted and discussed in Sect. 5, and the conclusions are drawn in Sect. 6.

\section{Data set}

In the present study the soft X-ray and hard X-ray bursts are compared using the SXR data from the Geostationary Operational Environmental Satellites (GOES) and the HXR data from the Burst and Transient Source Experiment (BATSE) aboard the Compton Gamma-Ray Observatory (CGRO). The $\mathrm{X}$-ray sensor aboard GOES consists of two ion chamber detectors, which provide whole-sun X-ray fluxes in the 0.05-0.4 and $0.1-0.8 \mathrm{~nm}$ wavelength bands. A description of the GOES ion chambers can be found in Donelly \& Unzicker (1974) and Garcia (1994). BATSE is a whole-sky HXR flux monitor that consists of eight large-area wide-field detectors, placed on the corners of the CGRO spacecraft. From each of eight detectors there are hard X-ray data in four energy channels, 25-50, 50-100, 100-300 and $>300 \mathrm{keV}$, obtained with a time resolution of about $1 \mathrm{~s}$. Technical characteristics of the BATSE instrument and its application to solar flare studies are described in Fishman et al. $(1989,1992)$ and Schwartz et al. (1992).

We utilize the 1-min averaged GOES soft X-ray data in the $0.1-0.8 \mathrm{~nm}$ wavelength band as listed in the flare compilation of the Solar Geophysical Data (SGD, $\mathrm{ftp}$ ://ftp.ngdc.noaa.gov/STP/SOLAR_DATA/SOLAR_FLARES/), and the hard X-ray data from the BATSE Solar Flare Catalog, archived in the Solar Data Analysis Center (SDAC) at NASA/Goddard Space Flight Center (GSFC, ftp://umbra.nascom.nasa.gov/pub/batse/). In the BATSE Flare Catalog the start, maximum, and end time of an event are listed with an accuracy of $1 \mathrm{~s}$. The peak count rate and the total count rates are background subtracted for the flux below $100 \mathrm{keV}$. For the SXR events observed by GOES we used the flux just before the flare start for the background subtraction. The analysis was carried out for the period from January 1997 to June 2000 (when CGRO was deorbited), for which 6947 SXR events and 2738 HXR events are reported.

Due to the lack of spatial information, the determination of related SXR/HXR flares is exclusively based on temporal coincidence. To be identified as corresponding events, we demand that the start time difference between a SXR and a HXR event does not exceed $10 \mathrm{~min}$. To avoid as much as possible any incidental assignment, we applied the following refinements. All SXR (HXR) events that overlap in time with any other SXR (HXR) event are excluded. Moreover, events for which a multiple assignment is possible (e.g., one SXR event can be related to two different HXR events by the start time criterion) are excluded from the analysis. Applying these criteria, we obtained 1404 events that were observed in both SXR and HXR emissions. This data set was reduced to a final set of 1114 SXR/HXR events, after excluding those events with missing SXR background flux data.

The characteristic times, which are under study, are the peak time of the SXR emission and the end time of the HXR emission. It has to be noted that the end of a HXR event is difficult to determine, whereas the SXR peak time is a rather well defined quantity (at least within the given precision of $1 \mathrm{~min}$ ). So, it has to be kept in mind that there is a statistical error, in particular related to the HXR end time, which introduces also a statistical error on the HXR fluence data. Thus, a scatter in the relevant figures has to be expected in addition to the scatter caused by different physical conditions in flares. Moreover, we stress that for flares of low SXR or low HXR intensities, the peak flux and fluence data are affected by observational selection/sensitivity effects, which has to be considered when interpreting the data. In each of the figures, the estimated threshold ranges for the relevant parameters are indicated. See Lee et al. (1993) for methods of handling these selection effects.

\section{The Neupert effect and the chromospheric evaporation model}

The Neupert effect, as it is commonly stated in the literature, can be expressed as (e.g., Lee et al. 1995)

$F_{\mathrm{P}, \mathrm{SXR}}=k \cdot \mathcal{F}_{\mathrm{HXR}}$ 
whereas $F_{\mathrm{P}, \mathrm{SXR}}$ denotes the SXR peak flux, and

$\mathcal{F}_{\mathrm{HXR}}=\int_{t_{0}}^{t_{0}+D} F_{\mathrm{HXR}}(t) \mathrm{d} t$

the HXR fluence, i.e. the HXR flux, $F_{\mathrm{HXR}}(t)$, integrated over the event duration $D$ starting at time $t_{0}$. The coefficient $k$ depends on several factors, as, for instance, the magnetic field geometry and the viewing angle, and thus may vary from flare to flare (see Lee et al. 1995). However, if $k$ does not depend systematically on the flare intensity, then from the Neupert effect stated in Eq. (1), a linear relationship is expected to exist between the SXR peak flux and the HXR fluence.

On the basis of flare frequency distributions, Lee et al. (1993, 1995) and Veronig et al. (2002a) found inconsistencies with the linearly formulated Neupert effect as given in Eq. (1). If $k$ does not depend systematically on the flare intensity, then the HXR fluence and the SXR peak flux distributions should have the same shape, in particular they should be described by the same power-law index. However, the power-law index derived from HXR fluence distributions, $1.4 \lesssim \alpha \lesssim 1.6$ (cf. Lee et al. 1993, and references therein) is distinctly smaller than those obtained for SXR peak flux distributions, $1.8 \lessgtr \alpha \lesssim 2.0$ (cf. Veronig et al. 2002a, and references therein).

A possible explanation for this discrepancy is that the HXR and SXR emissions are not directly indicative for the energies involved, in the sense that the energies are not simply linearly related to the emissions. As emphasized in Lee et al. (1995), the Neupert effect interpreted as a consequence of electron-beamdriven chromospheric evaporation, should exist not necessarily between the X-ray emissions but between the energies. In the frame of the electron-beam-driven evaporation model, the $\mathrm{HXR}$ emission is a measure of the rate of energy deposition by accelerated electrons and the SXR emission is a measure of the total energy contained in the plasma heated by thermalization of the same electrons. Thus, the energy deposited by the nonthermal electrons, $\epsilon_{\mathrm{e}^{-}}$, should be equal to the maximum thermal energy contained in the plasma that is heated by this electron population, $\epsilon_{\mathrm{th} \text { max }}$, i.e.

$\epsilon_{\mathrm{e}^{-}}=\epsilon_{\mathrm{th}, \max } \cdot$

Since the relation between the energies and the X-ray emissions is not necessarily linear, Eq. (3) is compatible with the possibility that the factor $k$ may be a function of the flare intensity, violating a linear interpretation of the Neupert effect formulated for the X-ray emissions (Eq. (1)). Thus, a dependence of $k$ on the flare intensity does not necessarily indicate that the Neupert effect formulated for the energies (Eq. (3)), i.e. the electron-beam-driven chromospheric evaporation model, is violated. Results reported in several recent papers indicate that $k$ indeed might depend on the flare intensity, suggesting that the amount of SXR emission per HXR electron may differ for small and large flares (Jim McTiernan, private communication). Feldman et al. (1996) and Garcia (2000) report that the observed SXR temperature tends to increase with flare intensity. On the other hand, as shown by McTiernan et al. (1999), consistency of the observed HXR and SXR emission with the Neupert effect depends on the temperature response of the SXR detector used. The Neupert effect is more commonly associated with SXR emission at high than low temperatures (McTiernan et al. 1999), which might be a further indication for an interdependence of $k$ with the flare intensity. Furthermore, Tomczak (1999) reports that the photon spectral index of the measured HXR emission as well as the relative SXR - HXR productivity depend systematically on the flare intensity. However, no systematic dependence of the photon spectral index on the flare intensity was found by Dennis (1985), especially for gradual flares.

Another possibility is that the Neupert effect, formulated for the X-ray emissions (Eq. (1)) as well as for the more fundamental relationship between the thermal and nonthermal energies (Eq. (3)), does not hold for the bulk of flares but maybe only for a specific subset. Most observational evidence for the Neupert effect is provided for large and impulsive flares (e.g., Dennis \& Zarro 1993; McTiernan 1999). Any deviation from the Neupert effect, in principle, means that the hot SXR emitting plasma is not heated exclusively by thermalization of the accelerated electrons that are responsible for the HXR emission (Dennis \& Zarro 1993; Lee et al. 1993). In this case, an additional energy term has to be added on the left hand side of Eq. (3).

Several attempts have been made to investigate the relationship between energies associated with HXR and SXR bursts estimating the total energy contained in precipitating electrons from measured HXR spectra and the thermal energy of the heated plasma from SXR measurements (e.g., Tanaka et al. 1982; Antonucci et al. 1984; Wu et al. 1986; Starr et al. 1988). However, as pointed out by Wu et al. (1986), neither the thermal nor the nonthermal energy can be estimated to better than an order of magnitude. The uncertainties of the nonthermal energy calculations from the HXR measurements are basically due to the fact that the low energy cut-off in the electron spectrum is unknown and may vary from flare to flare. Furthermore, the low energy cut-off may also vary during a given flare (Gan et al. 2002). The thermal energy calculations are uncertain primarily due to the estimates of the volume, filling factor and density of the emitting plasma.

In the following, we analyze the Neupert effect comparing the directly observable X-ray emissions. One aspect is the analysis of the relative timing of corresponding SXR and HXR events. The interpretation of the Neupert effect in the frame of the electron-beam-driven evaporation model implies that the end of the HXR burst should be coincident with the maximum of the SXR emission: when the electron input stops, the HXR emission also has to stop, and the SXR emission does not further increase. In principle, the HXR and SXR emission should also start at the same time. However, in more than $90 \%$ of flares the SXR emission starts before the HXR emission by at least several minutes (Veronig et al. 2002b). This may be indicative of a thermal preheating phase prior to the impulsive electron acceleration or it may be related to the sensitivity threshold of the hard X-ray detectors (see also Dennis 1988). Thus, we do not incorporate an investigation of the HXR - SXR start time differences.

The other aspect is to analyze the HXR fluence - SXR peak flux relationship. A simple prediction of the Neupert effect is 
that there should be a high correlation among these two parameters, even if the relationship is not linear. In particular, we will also investigate the factor $k$ as a function of the flare intensity. Additionally, making use of the results from the timing analysis, different subsets of events will be extracted, which are likely compatible/incompatible with the Neupert effect regarding their temporal behavior. Differences in the HXR fluence SXR peak flux relationship between these subsets may help in understanding the role of $k$ in the frame of the Neupert effect.

\section{Analysis and results}

\section{1. $S X R-H X R$ timing}

For each event we determined the difference of the peak time of the SXR emission, $t_{\mathrm{SXR}, \mathrm{P}}$, and the end time of the HXR emission, $t_{\mathrm{HXR}, \mathrm{E}}$ :

$\Delta t=t_{\mathrm{SXR}, \mathrm{P}}-t_{\mathrm{HXR}, \mathrm{E}} \cdot$

Furthermore, the time differences were normalized to the duration $D$ of the respective HXR event:

$\Delta t_{\text {norm }}=\frac{\Delta t}{D}$.

The normalized time differences are of particular interest when the timing behavior of long-duration flares is considered. Such events may show considerable time differences but these may be small compared to the overall duration of the event. As intense flares tend to be of longer duration than weak flares (see e.g., Crosby et al. 1998; Veronig et al. 2002a), applying a criterion exclusively based on absolute time differences will act selectively on intense flares. From the present data set we obtain a cross-correlation coefficient (calculated in logarithmic space), $r=0.47$ for the SXR peak flux and SXR flare duration, and $r=0.55$ for the HXR peak flux and HXR event duration, indicating a distinct correlation between the intensity of an event and its endurance. In general, the duration of the HXR emission of a flare is much shorter than that of the SXR emission. From the present data set, we derive a median duration (given with $95 \%$ confidence interval) of $1.9 \pm 0.2$ min for the HXR events and $12.0 \pm 0.5 \mathrm{~min}$ for the SXR events.

Figure 1 shows the histogram of time differences derived for 1114 SXR/HXR bursts, in absolute values (top panel) and normalized to the HXR event duration (bottom panel). As the HXR times are given with an accuracy of $1 \mathrm{~s}$ and the SXR times with an accuracy of $1 \mathrm{~min}$, we cannot expect to obtain reliable time differences $\lesssim 1 \mathrm{~min}$. Thus, for the derivation of the time differences, the HXR times have been rounded to minutes. The histogram of the $\Delta t$ uses a bin size of $1 \mathrm{~min}$. In the histogram of the $\Delta t_{\text {norm }}$, a bin size of 0.5 units of the HXR duration is used. The HXR events have a median duration of $1.9 \mathrm{~min}$, thus on average a time difference of 0.5 units in the normalized representation can be considered to roughly correspond to a time difference of $1 \mathrm{~min}$ for the absolute values.

Figure 1 clearly reveals that both representations of the SXR - HXR time difference have its mode at zero. Almost half of the events (49\%) lie within the range $|\Delta t| \leq 1 \mathrm{~min}$, and $65 \%$ within the range $|\Delta t| \leq 2 \mathrm{~min}$. For the normalized differences
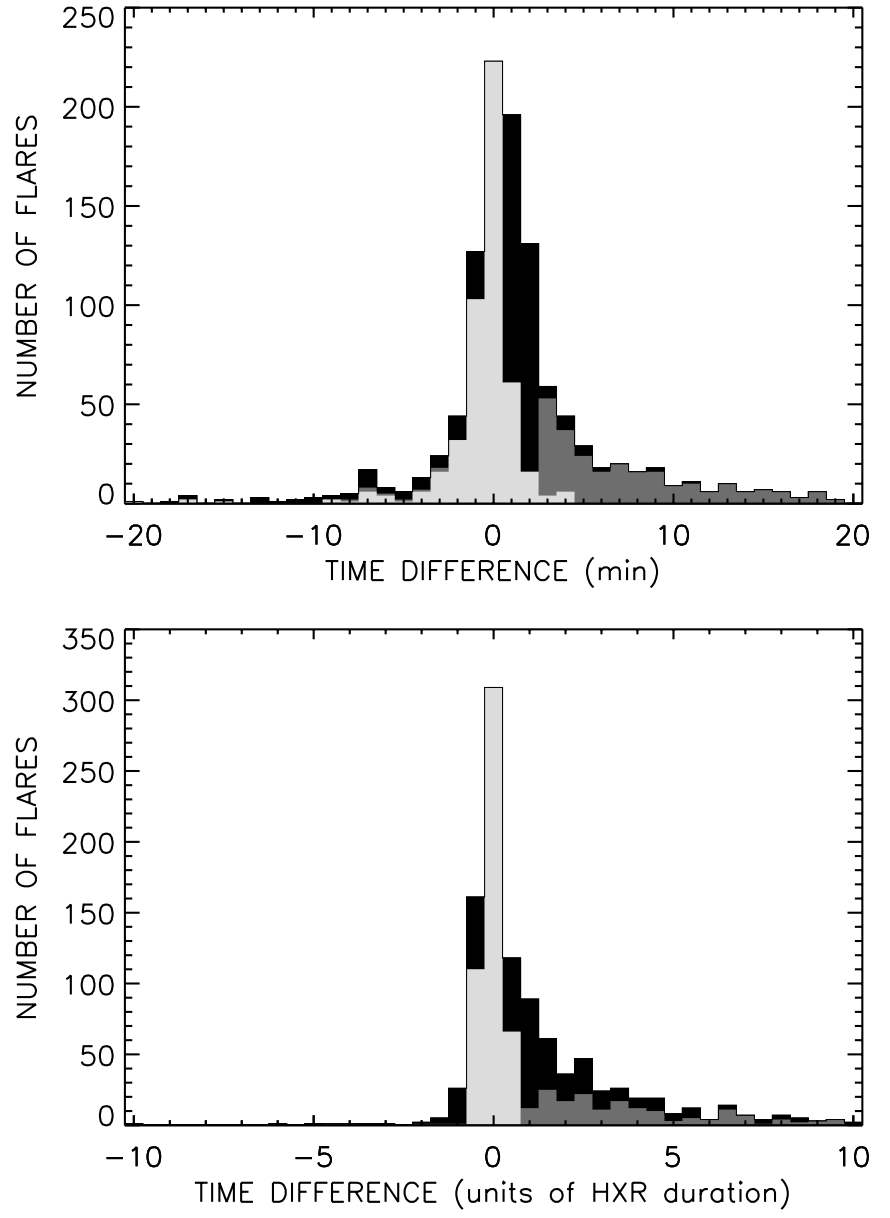

Fig. 1. Histogram of the difference of the SXR maximum and HXR end time, given in absolute values (top panel) and normalized to the HXR event duration (bottom panel). Positive values indicate that the maximum of the SXR emission occurs after the end of the HXR emission, negative values vice versa. The shading refers to different samples of events, which are compatible with the timing expectations of the Neupert effect (light grey, set 1), strongly incompatible (dark grey, set 2), or lie in between these two extremes (black). For further explanations see Sect. 4.3.

we obtain that $44 \%$ lie within the range $\left|\Delta t_{\text {norm }}\right| \leq 0.5$ units, and $59 \%$ within $\left|\Delta t_{\text {norm }}\right| \leq 1$ unit. This outcome suggests that certainly a considerable part of the events shows a good agreement with the expectations from the Neupert effect regarding the relative timing of the SXR and HXR emission. Furthermore, the histograms in Fig. 1 show that there are more events, for which the SXR maximum takes place after the HXR end (56\%) than vice versa $(24 \%) .20 \%$ of the events do not show a distinguishable time difference, i.e. the SXR maximum and the HXR end take place within $1 \mathrm{~min}$. This asymmetric behavior is particularly evident for the distribution of the normalized time differences.

\subsection{SXR peak flux - HXR fluence relationship}

Figure 2 shows the scatter plot of the SXR peak flux versus the HXR fluence for the complete sample, clearly revealing an increase of $F_{\mathrm{P}, \mathrm{SXR}}$ with increasing $\mathcal{F}_{\mathrm{HXR}}$. It can also be 


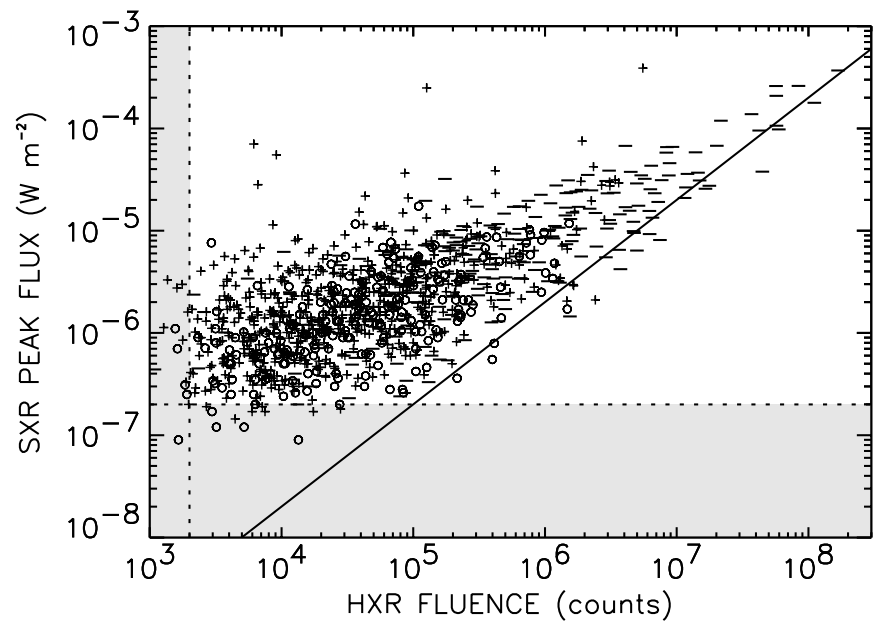

Fig. 2. Scatter plot of the SXR peak flux versus the HXR fluence for the complete sample. The vertical dashed line indicates the HXR fluence threshold, the horizontal dashed line the SXR peak flux threshold. The regions that lie outside the respective detection limits are grey shaded. Moreover, we have indicated the sign of the time difference between SXR peak and HXR end for each single event: "+" symbols denote events with positive, "-" symbols events with negative, "o" symbols events with zero time difference. The straight line indicates a line of constant $k$, i.e. $F_{\mathrm{P}, \mathrm{SXR}}=2 \times 10^{-12} \cdot \mathcal{F}_{\mathrm{HXR}}$.

inferred from the figure that the slope is not constant over the whole range but that it is larger for large HXR fluences than for small ones. We stress that the slope at small fluences might be affected by missing events with small SXR peak fluxes, and thus appear flatter than it is in fact. The SXR threshold is basically caused by the GOES flare listings, which generally do not embrace flares weaker than B class ${ }^{1}$. The HXR fluence cut-off arises due to the sensitivity limits of the HXR detectors as well as due to constraints of the exact start/end time determination for very short or weak flares.

In Fig. 2 (note that the same holds for Figs. 3-9) we have indicated the estimated thresholds of event detection by grey shading, in order to visualize which ranges might be biased by cut-off effects. From this representation it is evident that for the range $\mathcal{F}_{\text {HXR }} \gtrsim 2 \times 10^{5}$ counts, the scatter plot is not biased by the sensitivity thresholds.

The sign of the time difference between SXR peak and HXR end time of an event is indicated by different plot symbols. "+" symbols represent events with $\Delta t>0$, "-" symbols events with $\Delta t<0$. Events that do not show a distinguishable time difference, i.e. the SXR peak and HXR end take place within $1 \mathrm{~min}$, are indicated by "o" symbols. In each of the figures a line of constant $k$ is overplotted, choosing $k=2 \times 10^{-12} \mathrm{~W} \mathrm{~m}^{-2}$ counts $^{-1}$, which can be considered as an estimate of $k$ for the largest flares.

Figure 3 shows the SXR peak flux - HXR fluence relationship separately for the events with $\Delta t>0, \Delta t<0$ and $\Delta t=0$. The figure clearly reveals an interdependence between the importance of an event and the sign of the time difference. Basically all large flares belong to the group of events with

\footnotetext{
1 Defined by a SXR peak flux without background subtraction of $10^{-7} \mathrm{~W} \mathrm{~m}^{-2}$.
}

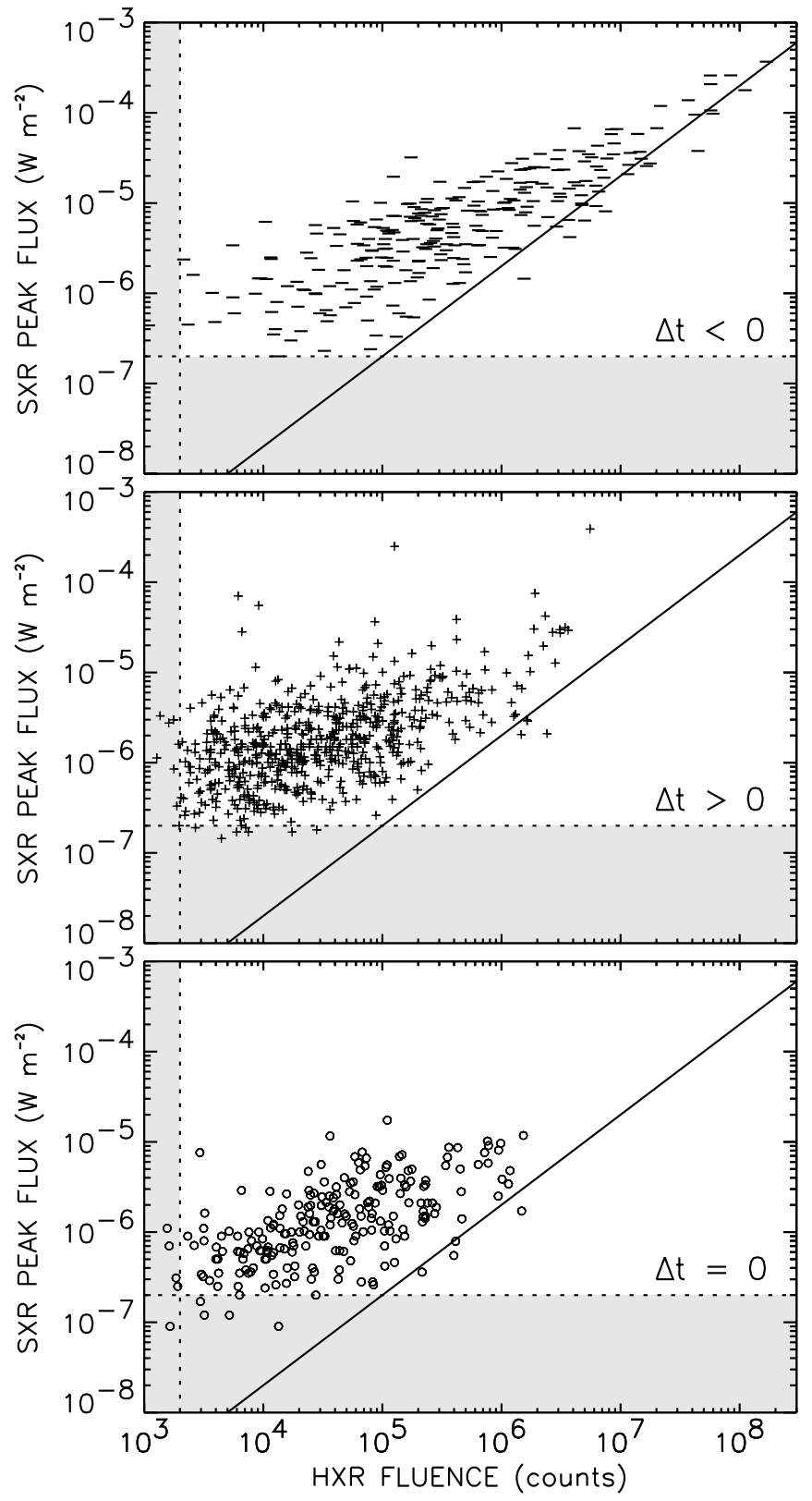

Fig. 3. Scatter plot of the SXR peak flux versus the HXR fluence separately for events with negative (top panel), positive (middle panel) and zero (bottom panel) time difference. The same line of constant $k$ $\left(=2 \times 10^{-12}\right)$ is shown in each case.

$\Delta t<0$, i.e. the SXR peak occurs before the HXR end. On the other hand, this group covers distinctly fewer weak flares than the group of events with $\Delta t>0$.

From Figs. 2 and 3 it can be seen that, for very large fluences, the slope of the SXR peak flux versus HXR fluence approaches the value of 1 . This phenomenon shows up even clearer in Fig. 4, in which the factor $k$ as function of the HXR fluence is plotted. In general, $k$ is decreasing for increasing $\mathcal{F}_{\text {HXR }}$. Yet, for large fluences, $k$ becomes nearly constant, indicating an approximately linear relationship between the SXR peak flux and the HXR fluence for the most intense events. We have applied a linear least-squares fit to the data shown in Fig. 4 in the range $\mathcal{F}_{\text {HXR }}>10^{6}$ counts and $\mathcal{F}_{\text {HXR }}>10^{7}$ counts. 


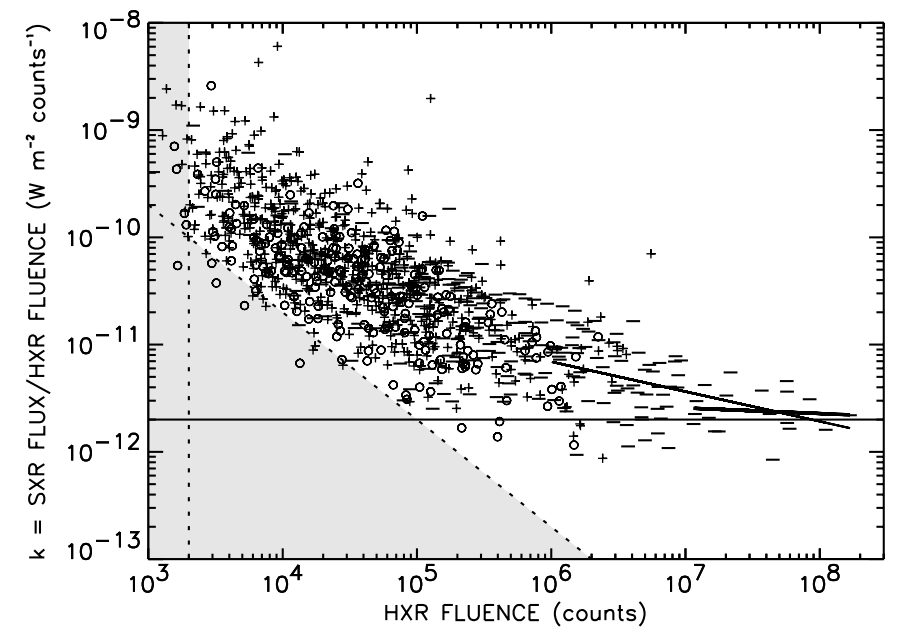

Fig. 4. Scatter plot of the factor $k$, i.e. SXR peak flux divided by HXR fluence, versus HXR fluence for the complete sample. The same line of constant $k\left(=2 \times 10^{-12}\right)$ is shown as in Fig. 2. Furthermore, we have plotted linear least-squares fits to the data, derived in the range $\mathcal{F}_{\mathrm{HXR}}>10^{6}$ counts and $\mathcal{F}_{\mathrm{HXR}}>10^{7}$ counts, respectively.

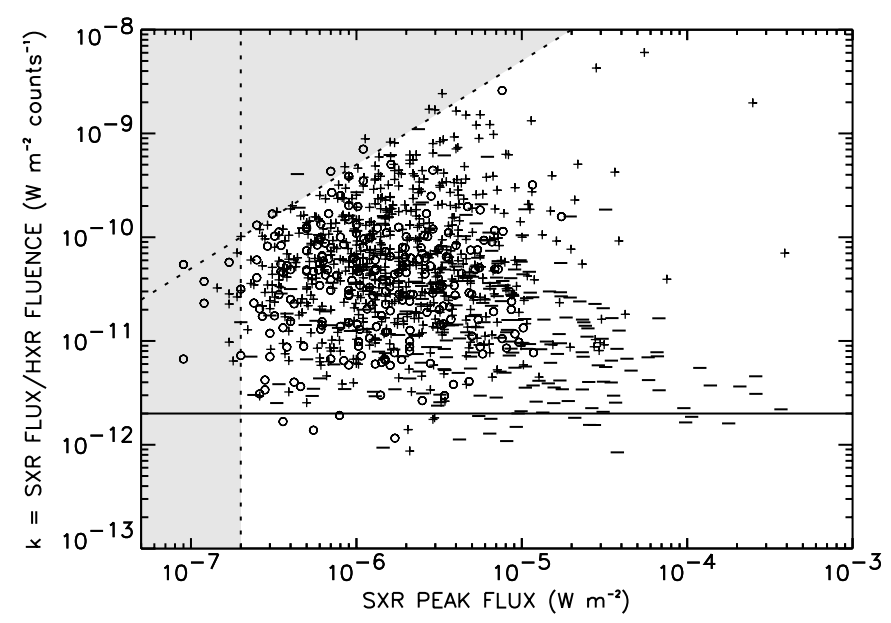

Fig. 5. Scatter plot of the factor $k$ versus SXR peak flux for the complete sample. The same line of constant $k\left(=2 \times 10^{-12}\right)$ is shown as in Fig. 2.

The obtained slopes give $b=-0.28 \pm 0.06$ and $b=-0.05 \pm 0.09$, respectively. The least-squares fits are indicated in the figure by straight lines.

In Fig. 5, $k$ as function of the SXR peak flux is plotted, revealing a very large scatter and a less distinct behavior than for the HXR fluence. However, for events with negative time differences (indicated by "-" symbols), $k$ shows a tendency to decrease with increasing SXR peak flux, approaching an almost constant $k$ for very large peak fluxes (note that this phenomenon is not biased by threshold effects). Events with positive time difference reveal a tendency to increase with increasing SXR peak flux. However, it cannot be excluded that this increasing behavior is biased by selection effects, missing events with small SXR fluxes.

We obtain a high cross-correlation coefficient, $r=0.71$, for the SXR peak flux and HXR fluence relationship. (All cross-correlation coefficients are determined in log-log space.)
This coefficient is higher than those for the SXR peak flux and HXR peak flux, $r=0.57$. This indicates that the correlation is primarily due to the HXR fluence - SXR peak flux relationship, as predicted from the Neupert effect, and not, e.g., due to the fact that flares with high HXR peak fluxes also tend to have intense SXR counterparts. However, since the fluence of an HXR event can be approximated by the product of peak flux and event duration (e.g., Lee et al. 1995), it is expected that the SXR peak flux - HXR fluence correlation is reflected also in a distinct correlation among the SXR and HXR peak fluxes. The derived cross-correlation coefficients are very similar to those reported by Wu et al. (1986) and Starr et al. (1988), who analyzed selected samples of HXR/SXR bursts.

Finally, it is important to note that the HXR fluence - SXR peak flux correlation is higher for the events with negative time differences, $r=0.82$, than for the events with positive time differences, $r=0.54$ (see also Table 1 ).

\subsection{SXR peak flux - HXR fluence analysis combined with the relative timing}

On the basis of the relative timing of the SXR peak and the HXR end, we extracted two subsets of events. The events of set 1 are roughly consistent with the timing expectations from the Neupert effect, and the events of set 2 are inconsistent with it. The two sets are defined by the following conditions:

Set 1: $(|\Delta t|<1 \mathrm{~min})$ or $\left(\left|\Delta t_{\text {norm }}\right|<0.5\right.$ unit $)$,

Set 2: $(|\Delta t|>2 \mathrm{~min})$ and $\left(\left|\Delta t_{\text {norm }}\right|>1.0\right.$ unit $)$.

Out of the 1114 corresponding HXR and SXR flares, 485 (44\%) events fulfilled the timing criterion of set $1 ; 270$ events (24\%) belong to set $2 ; 359$ events $(32 \%)$ are neither attributed to set 1 nor to set 2 .

The applied conditions represent a combination of absolute and normalized time differences in order to avoid as much as possible any a priori interdependence with the flare duration and/or flare intensity. For example, the first part of the condition defining set 1 , which is based on absolute differences, is most likely to be fulfilled in flares of short duration. On the other hand, the second part, based on normalized differences, checks for consistency with the Neupert effect in the case of long-duration flares. The exact values chosen (1 min, $2 \mathrm{~min}$; 0.5 unit, 1.0 unit) are, of course, somewhat arbitrary. However, we stress that the two sets are defined as non-adjoining, i.e. there is a significant fraction of events (about one third) that are not used. We have also repeated the respective parts of the analysis with modified values. This changed the number of events covered by the respective sets but the results were qualitatively the same, as long as the applied changes were not too large and the data sets were not reduced too much.

In the following, we analyze in detail the HXR fluence SXR peak flux relationship separately for both subsets in order to find out whether there are distinct differences besides the temporal behavior. Figure 6 shows the scatter plot of the SXR peak flux versus the HXR fluence separately for set 1 and set 2 . Indeed, the two sets reveal very different characteristics. Set 1 contains many more large events and shows a steeper increase 

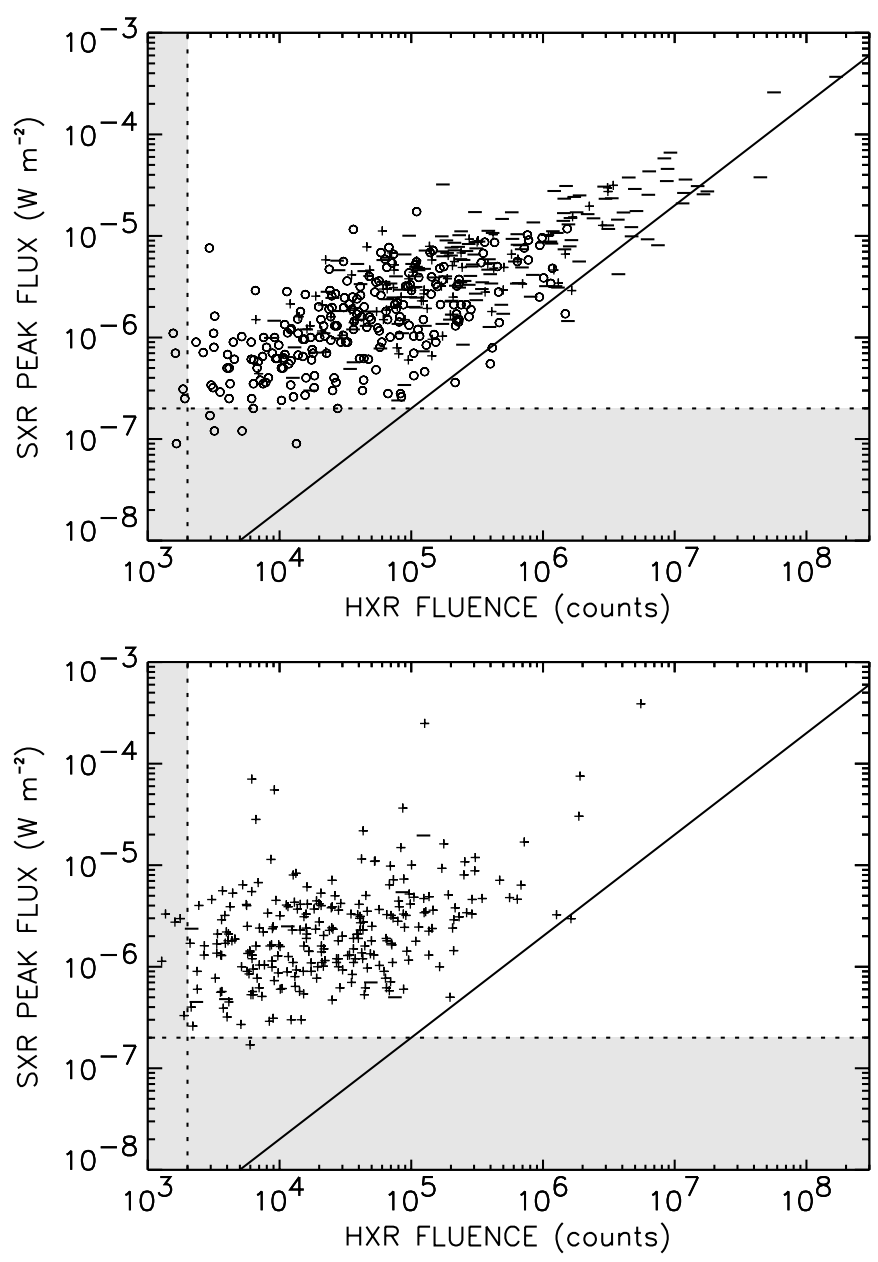

Fig. 6. Scatter plot of the SXR peak flux versus the HXR fluence separately plotted for set 1 (top panel) and set 2 (bottom panel).

of $F_{\mathrm{P}, \mathrm{SXR}}$ with increasing $\mathcal{F}_{\mathrm{HXR}}$ than set 2 . Moreover, set 1 contains many more events with negative than positive time difference, although their absolute number is much smaller (cf. Table 1). The subdivision of set 1 with regard to the time differences gives: $\Delta t<0: 36 \%, \Delta t>0: 18 \%, \Delta t=0: 46 \%$. Almost all events of set $2(97 \%)$ are characterized by $\Delta t>0$, i.e. increasing SXR emission while the HXR emission already stopped. Obviously, considering the chosen criterion, it is not expected that set 2 contains events with $\Delta t \leq 0$ (see also the discussion in Sect. 5.1).

Furthermore, for small fluences, the events belonging to set 2 have a larger SXR peak flux at a given HXR fluence than do those of set 1 , indicating an "excess" of SXR emission with respect to set 1 . For instance, the median of the SXR peak flux, determined in the range $\mathcal{F}_{\mathrm{HXR}}<2 \times 10^{4}$ counts, gives $(6.5 \pm 1.0) \times 10^{-7} \mathrm{~W} \mathrm{~m}^{-2}$ for the events of set 1 , and $(16.0 \pm 3.2) \times 10^{-7} \mathrm{~W} \mathrm{~m}^{-2}$ for the events of set 2 . Note that this difference is not biased due to missing events below the thresholds, as this should affect both sets in the same way.

The cross-correlation coefficients derived separately for the subsets reveal that the correlation among the SXR peak flux and HXR fluence is much more pronounced for the events of set 1 , $r=0.78$, than those of set $2, r=0.41$. A similar trend holds
Table 1. Cross-correlation coefficients derived for the SXR peak flux and HXR fluence, and the SXR peak flux and HXR peak flux. The correlations $(r)$ are listed for the total of events as well as separately for events belonging to set $1 /$ set 2 and events with negative/positive time difference.

\begin{tabular}{lrrrrr}
\hline \hline & All & Set 1 & Set 2 & $\Delta t<0$ & $\Delta t>0$ \\
\hline No. of events & 1114 & 485 & 270 & 269 & 622 \\
$(\%)$ of total & 100 & 44 & 24 & 24 & 56 \\
$r\left(F_{\mathrm{P}, \text { SXR vs. }} \mathcal{F}_{\mathrm{HXR}}\right)$ & 0.71 & 0.78 & 0.41 & 0.82 & 0.54 \\
$r\left(F_{\mathrm{P}, \text { SXR }}\right.$ vs. $\left.F_{\mathrm{P}, \mathrm{HXR}}\right)$ & 0.57 & 0.58 & 0.28 & 0.68 & 0.36 \\
\hline
\end{tabular}
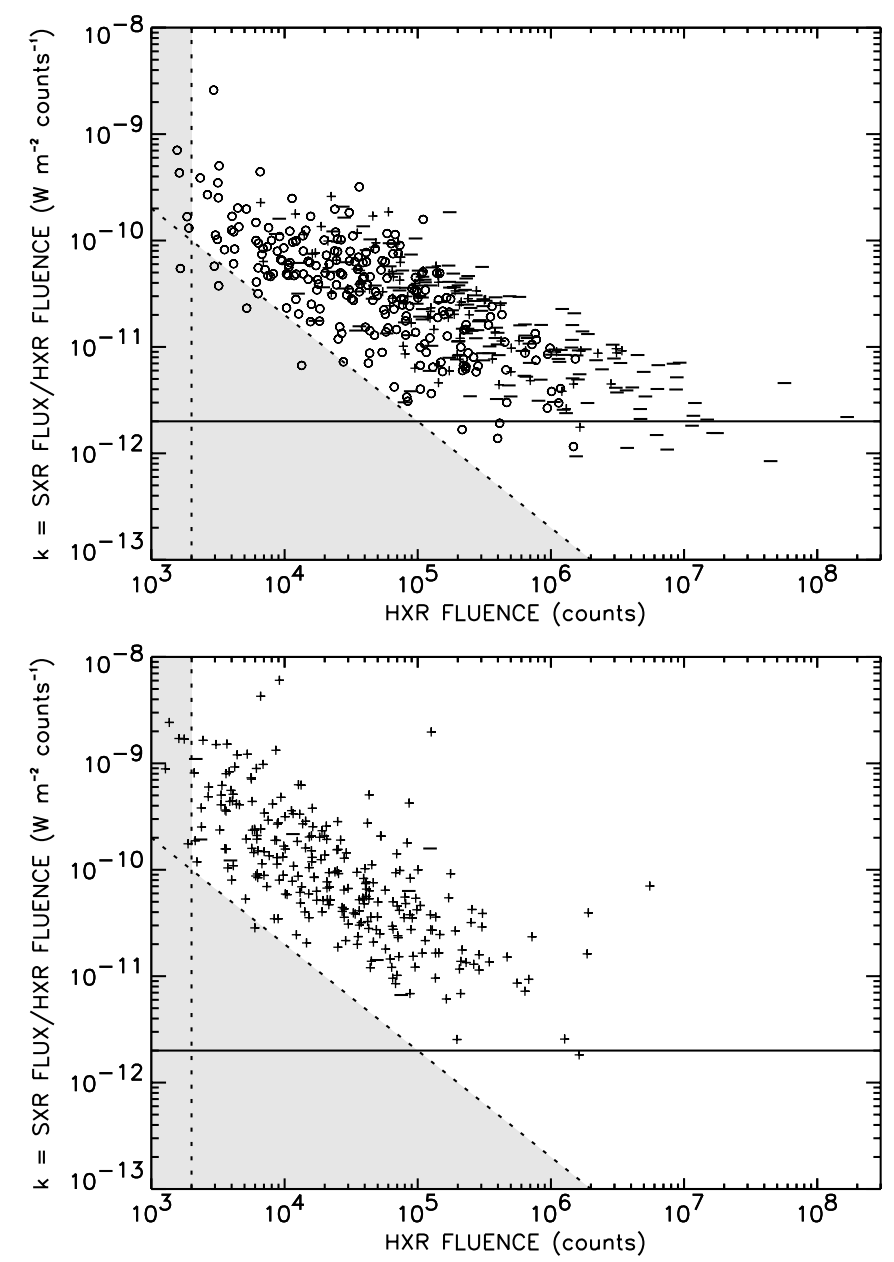

Fig. 7. Scatter plot of the factor $k$ versus the HXR fluence for set 1 (top panel) and set 2 (bottom panel).

also for the SXR and HXR peak flux relationship. The results of the cross-correlation analysis are summarized in Table 1.

In Figs. 7-9, the factor $k$ is plotted as function of the HXR fluence, the HXR peak flux and the SXR peak flux, respectively. Figure 7 reveals that $k$ is a distinct function of the HXR fluence. However, the specific behavior is different for the two sets. For set 2, the mean $k$ is a rather monotonically decreasing function of the HXR fluence, with a slope $b \sim-1.1$. For set 1 , $k$ shows a different behavior. For small and medium fluences, $k$ decreases with a slope smaller than that of set $2, b \sim-0.8$; 

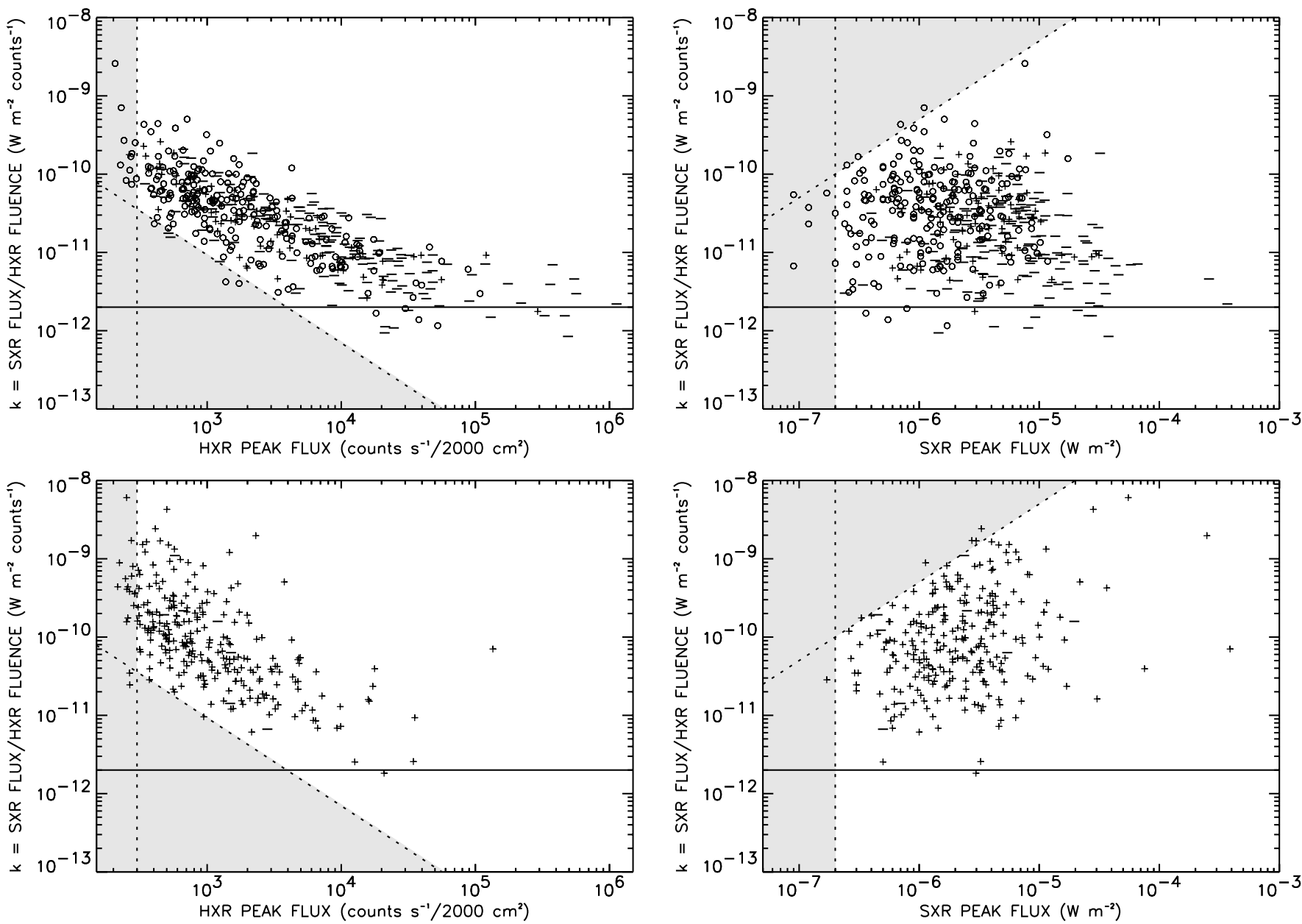

Fig. 8. Scatter plot of the factor $k$ versus the HXR peak flux for set 1 (top panel) and set 2 (bottom panel).

for large fluences, $k$ becomes almost constant, i.e. it is approximately independent of the HXR fluence.

A quite similar overall behavior is found for the dependence of $k$ on the HXR peak flux (Fig. 8), revealing also a very distinct relationship between these parameters. In Fig. 9, $k$ as function of the SXR peak flux is plotted. Both sets reveal a large scatter. For set 1, a poor anti-correlation exists between these two quantities, indicating that $k$ only weakly depends on the SXR peak flux $(r \sim-0.2)$. For set $2, k$ reveals a weak positive correlation with the SXR peak flux $(r \sim 0.3)$. However, this increase of $k$ with increasing SXR peak flux might be an artifact caused by threshold effects.

We have applied least-squares fits in the form $\log (k)=$ $a+b \cdot \log (A)$ for various SXR and HXR parameters, denoted here as $A$. Since we are interested in the functional dependence between $k$ and the respective HXR and SXR parameters, both variables should be treated symmetrically in the fit procedure. This symmetric treatment is not satisfied by ordinary leastsquares regression of an dependent variable $y$ on an independent variable $x$. Isobe et al. (1990) have shown that regression using the bisector of the two ordinary least-squares regression $y$ on $x$ and $x$ on $y$ is the most suitable fitting method, if the goal is to determine the underlying functional relationship between the

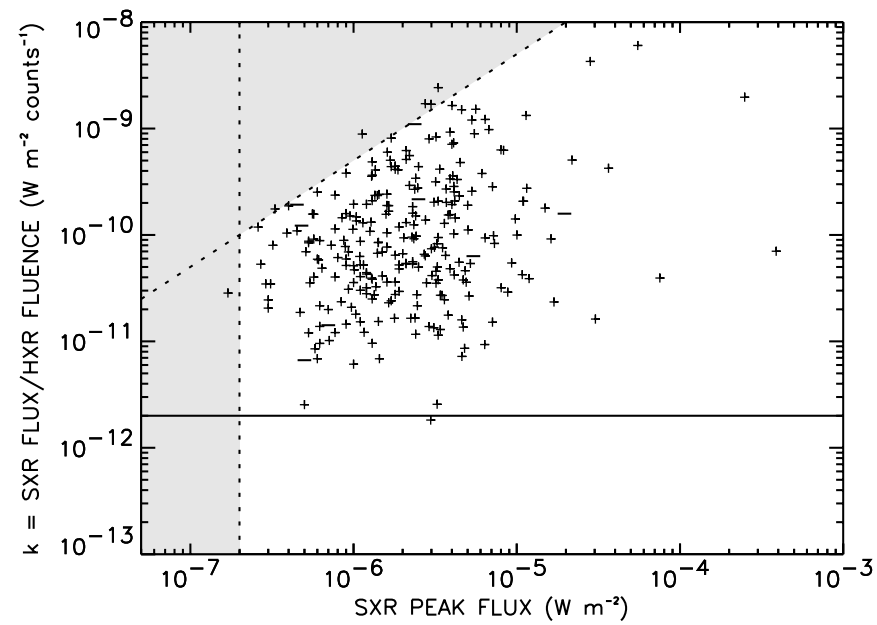

Fig. 9. Scatter plot of the factor $k$ versus the SXR peak flux for set 1 (top panel) and set 2 (bottom panel).

variables. Thus, we have applied the bisector fitting technique (for details see Isobe et al. 1990).

The results of the regression analysis are summarized in Table 2. In order to compare the respective fits of set 1 and set 2 , we determined the fits only in a range of values of the respective parameters (indicated in Table 2) in which both data sets cover a significant number of points. The table reveals that the characteristics of both sets are very different. In all cases, the difference of the fit parameters, derived separately from set 1 and set 2 are larger than the given uncertainties. (Note that due to the large scatter in the graphs of $k$ versus the SXR peak flux (Fig. 9), the respective fit parameters differ very much for different fitting techniques, and have to be taken with caution.)

\section{Discussion}

\subsection{SXR - HXR Timing}

The timing analysis shows that $44 \%$ of the events obey the chosen criterion indicative of the Neupert effect, i.e. the difference of the SXR peak time and the HXR end time is less than $1 \mathrm{~min}$ or less than 0.5 times the HXR duration, whereas $24 \%$ reveal a strong deviation. Obviously, this estimate depends on the chosen criteria. Nevertheless, the outcome suggests that a large 
Table 2. Outcome of the regression analysis of $k$ as function of the HXR fluence, the HXR peak flux and the SXR peak flux, determined separately for both sets. The fits are of the form $\log (k)=a+b \cdot \log (A)$. Additionally, the respective cross-correlation coefficients $r$ are listed.

\begin{tabular}{c|ccc|cccc|ccc}
\hline \hline \multicolumn{1}{c}{$A$} & $\begin{array}{c}\mathcal{F}_{\mathrm{HXR}}<3 \times 10^{5} \\
\text { (counts) }\end{array}$ & \multicolumn{4}{c}{$\begin{array}{c}F_{\mathrm{P}, \mathrm{HXR}}<1 \times 10^{4} \\
\left(\mathrm{counts} \mathrm{s}^{-1} / 2000 \mathrm{~cm}^{2}\right)\end{array}$} \\
\hline & $a$ & $b$ & $r$ & $a$ & $b$ & $r$ & \multicolumn{2}{c}{$\begin{array}{c}F_{\mathrm{P}, \mathrm{SXR}} \\
\left(\mathrm{W} \mathrm{m}^{-2}\right)\end{array}$} \\
Set 1: & $-6.52 \pm 0.16$ & $-0.84 \pm 0.03$ & -0.62 & $-7.09 \pm 0.13$ & $-1.05 \pm 0.04$ & -0.69 & $-16.18 \pm 0.11$ & $-0.99 \pm 0.02$ & -0.22 \\
Set 2: & $-5.33 \pm 0.21$ & $-1.09 \pm 0.05$ & -0.72 & $-5.44 \pm 0.24$ & $-1.54 \pm 0.08$ & -0.61 & $-3.34 \pm 0.38$ & $+1.18 \pm 0.07$ & +0.33 \\
\hline
\end{tabular}

fraction of the events reveal a timing behavior that is consistent with the Neupert effect; but there exists also a significant fraction of events that are incompatible with the Neupert effect.

Comparing the distributions of the absolute and normalized time differences (Fig. 1), it is noteworthy that a systematic difference shows up for negative differences, denoting events for which the SXR maximum occurs before the HXR end. Contrary to the distribution of the absolute time differences, the distribution of the differences normalized to the HXR duration reveals a sharp decline for negative differences, indicating that a substantial fraction of the events with negative differences are attributed to set 1 . Indeed, $65 \%$ of the events with $\Delta t<0$ belong to set 1 , whereas only $14 \%$ of the events with $\Delta t>0$ do.

In Fig. 10, we have plotted the absolute time difference between the SXR peak and the HXR end as function of the event duration, the HXR fluence and the SXR peak flux. Each of these plots has been carried out separately for events with positive and negative time differences, respectively. For the sample with negative time differences, we have overplotted the line of $|\Delta t|=D$, where $D$ is the HXR duration. For events that lie on the left hand side of this line, $D$ is smaller than the absolute value of the time difference, which can only mean that the SXR maximum occurs before the start of the HXR emission. Taking into account the given accuracy of $1 \mathrm{~min}$ for the SXR-HXR time differences, 14 events belong to this group (indicated by a $\times$-symbol in panels a-c in Fig. 10). In principle, there are two possible explanations for such events: either the respective SXR and HXR events are not causally related but occur incidentally within the applied 10-min start time window, or the given HXR duration is estimated too short due to the sensitivity limits of the HXR detectors. The second possibility is likely to apply for weak and short HXR events.

Comparing panels a and $\mathrm{d}$, it is noticeable that the sample with negative time differences covers very many events of long duration, say, $D \gtrsim 10$ min ( $~ 70$ events out of 269 ), whereas only a few events with long duration belong to the sample with positive time differences $(\sim 15$ events out of 622$)$. For the median HXR duration we obtain $\bar{D}=5.3 \pm 0.7 \mathrm{~min}$ for the events with $\Delta t<0$ and $\bar{D}=1.3 \pm 0.1 \mathrm{~min}$ for the events with $\Delta t>0$.

Li et al. (1993) have calculated time profiles of spatially integrated soft and hard X-ray emission from a thick-target electron-heated model, finding that in general the time derivative of the SXR time profile corresponds to the time profile of the HXR emission, as stated by the Neupert effect. However, for long-duration ("gradual") events they derived that this relationship breaks down during the decay phase of the HXR event, in that the maximum of the SXR emission occurs before the end
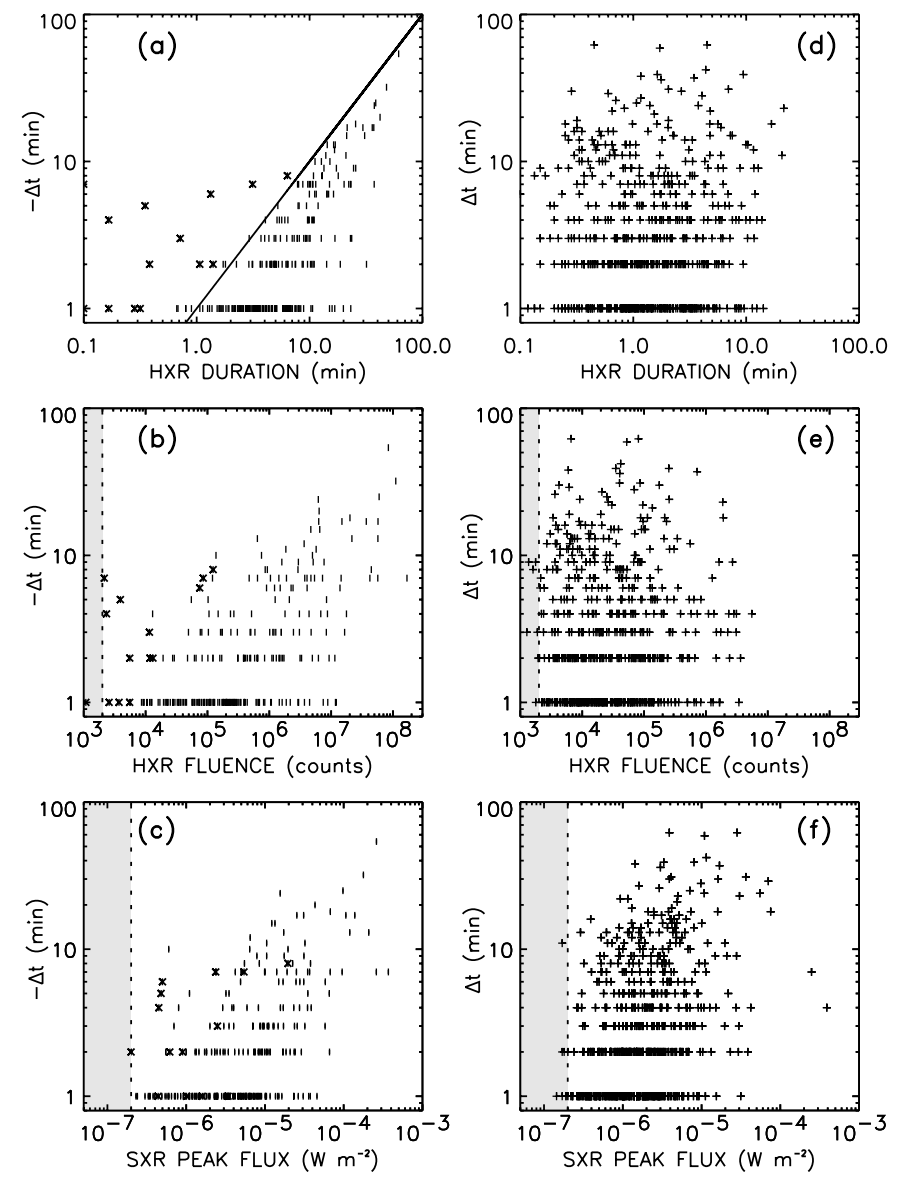

Fig. 10. We have plotted the absolute time difference between the peak of the SXR emission and the end of the HXR emission as a function of the event duration (top), HXR fluence (middle) and SXR peak flux (bottom), separately for the events with negative (panels a-c)) and positive (panels d-f)) time differences. The line in panel a) indicates $|\Delta t|=$ HXR duration.

of the HXR event. This phenomenon can be explained by the fact that the SXR emission starts to decrease if the evaporationdriven density enhancements cannot overcome the cooling of the hot plasma, which is likely to happen in gradual flares $(\mathrm{Li}$ et al. 1993).

Considering our observational findings together with the results from simulations by $\mathrm{Li}$ et al. (1993), it is very likely that most of the events with $\Delta t<0$ are consistent with the electron-beam-driven evaporation model. In particular, the very high correlation between the SXR peak flux and the HXR fluence obtained for these events, $r \sim 0.8$ (cf. Table 1), supports such interpretation. From the histogram of the normalized 
time differences (cf. Fig. 1, bottom panel), we obtain a median $\overline{\Delta t}_{\text {norm }}=-0.40 \pm 0.04$ for the events with $\Delta t<0$. Thus, on average, the instantaneous cooling of the SXR emitting plasma is dominating over the evaporation-driven energy supply for $\sim 0.4$ times the HXR duration during the decay phase of the HXR event, where the density is only slowly increasing.

For the events with $\Delta t<0$, there is a distinct correlation between the absolute value of $\Delta t$ and the SXR peak flux as well as the HXR fluence, $r \sim 0.6$ (cf. Fig. 10, panels b and c). This correlation is basically caused by the obvious correlation of the duration and the time difference (cf. panel a), $r \sim 0.6$. For the events with $\Delta t>0$, we find no correlation between the absolute value of $\Delta t$ and the event duration as well as the HXR fluence, $r<0.1$ (cf. panels $\mathrm{d}$ and e). However, a weak but significant correlation exists between $\Delta t$ and the SXR peak flux, $r=0.35$ (cf. panel f). The fact that the time difference and the HXR fluence of an event are not correlated but the time difference and the SXR peak flux are, might give indications that for these events an additional energy transport mechanism other than the HXR emitting electron beams is contributing to the SXR flux. On average, this contribution results in a comparatively higher SXR peak flux for large time differences than small ones. Under this assumption, we can estimate that, on average, the prolonged increase of the SXR emission takes place for $\sim 2$ times the HXR event duration $\left(\overline{\Delta t}_{\text {norm }}=2.00 \pm 0.32\right)$.

However, it has to be noted, that an extended heating beyond that due to nonthermal electron beams is not the only possible explanation for the events with $\Delta t>0$. Using data from the Yohkoh Soft X-ray Telescope (SXT) and Bragg Crystal Spectrometer (BCS), McTiernan et al. (1999) have shown that consistency with the Neupert effect depends on the SXR detector used. High-temperature plasma ( $T \gtrsim 16.5 \mathrm{MK})$ is more likely than low-temperature plasma to exhibit the Neupert effect. As shown by the authors, in several flares, the thermal energy derived from the low- $T$ component was still increasing after the end of the HXR emission, whereas the peak of the high- $T$ component occurred almost simultaneously with the HXR end. The curves of the total thermal energy peaked somewhere in between those of the low and high- $T$ components. Thus, an increase of the emission of low- $T$ plasma after the HXR end does not necessarily indicate an additional heating agent other than nonthermal electrons, but alternatively it might be due to cooling of the high- $T$ plasma (see McTiernan et al. 1999). The GOES $0.1-0.8 \mathrm{~nm}$ detector used in the present study also has a substantial response to low- $T$ plasma (Jim McTiernan, private communication). On the other hand, it is more sensitive to high- $T$ plasma than the SXT detectors used in the study by McTiernan et al. (1999).

So, due to this arguments of McTiernan et al. (1999), we cannot simply attribute all events with $\Delta t>0$ as inconsistent with the Neupert effect and the electron-beam-driven chromospheric evaporation model. Instead, we consider as inconsistent only flares, which show strong deviations from $\Delta t=0$, i.e. the events belonging to set 2 . From the present study, we can infer that about half of the events fulfill the timing criterion indicative for the Neupert effect (set 1), whereas about one fourth shows strong violations of the Neupert timing with prolonged SXR increase after the HXR end (set 2). This outcome is similar to the results from McTiernan et al. (1999), applying a measure that correlates the derivative of the SXR light curve with the HXR emission, that about half of the analyzed events are compatible with the Neupert effect, whereas for the other half an additional heating is indicated.

\subsection{Events of Set 2}

270 events ( $\sim 25 \%$ of the analyzed sample), belong to set 2 , i.e. they are characterized by strong deviations from the timing expectations of the Neupert effect, with the SXR maximum occurring distinctly after the HXR end. The fact that the SXR emission is still increasing although the HXR emission, i.e. the electron input, already stopped for more than 2 min and more than 1 unit of the HXR event duration, provides strong evidence that an additional agent besides the HXR emitting electrons is contributing to the energy input and prolonging the heating and/or evaporation. Possible sources invoked as additional heating agents are, for instance, thermal conduction (Zarro \& Lemen 1988; Yokoyama \& Shibata 2001; Czaykowska et al. 2001), accelerated protons (Simnett 1986; Plunkett \& Simnett 1994), plasma waves (Petrosian 1994; Lee et al. 1995) and DCelectric fields (McDonald et al. 1999).

Comparing the HXR fluence - SXR peak flux relationship for set 2 with that for set 1 (Fig. 6) clearly reveals that both sets have very different characteristics. Set 2 has many fewer large events, of, say, $\mathcal{F}_{\text {HXR }} \gtrsim 2 \times 10^{5}$ counts, than set 1 . For very small fluences, on average, the events of set 2 have larger SXR peak fluxes at given HXR fluences than those of set 1 . Moreover, for set 2 we obtain a distinctly smaller cross-correlation coefficient between the HXR fluence and the SXR peak flux, $r \sim 0.4$, than for set $1, r \sim 0.8$ (cf. Table 1). All these facts, the timing behavior, the higher SXR emission at a given HXR fluence for weak flares, and a low correlation between the HXR and SXR emission, provide evidence that the events of set 2, comprising $\sim 25 \%$ of the complete sample, are incompatible with the scenario, in which the hot plasma giving rise to the SXR emission is exclusively heated by thermalization of the electron beams responsible for the HXR emission.

\subsection{Events of Set 1}

What about the events belonging to set 1 ? We cannot straightforwardly conclude that these events are consistent with the Neupert effect and the electron-beam-driven evaporation model. The applied timing criterion presents a necessary condition for the Neupert effect (which is definitely violated by the events of set 2) but it is not a sufficient condition. In principle, two different possibilities can be distinguished. (i) The events of set 1 obey the Neupert effect formulated for the energies (Eq. (3)), i.e. the electron-beam-driven evaporation model, but there exists no simple linear proportionality between the X-ray emissions, i.e. the factor $k$ in Eq. (1) depends on the flare intensity. In this case, $k$ contains information on the relative productivity of soft X-ray emission per hard X-ray emission as a function of flare importance. (ii) Not only in the events belonging to set 2 but also in those of set 1 , an additional heating agent 
may be present. In this case, the heating of the SXR plasma due to this additional agent must have a similar timing to that of the nonthermal electrons; otherwise the timing expectations of the Neupert effect would be violated.

In the following section, we discuss the meaning of the factor $k$ from theoretical as well as observational considerations, based on the presumption that the electron-beam-driven chromospheric evaporation model is statistically valid for the events of set 1 in the sense of possibility (i), that they obey the Neupert effect. In Sect. 5.5, the results will be discussed with respect to possibility (ii), i.e. under the presumption of an additional heating agent.

\subsection{Relative productivity of $H X R$ and $S X R$ emission}

From theoretical considerations, it is evident that the relation between the involved thermal and nonthermal energies and the measured soft and hard X-ray emissions depend on a number of parameters. This may introduce a systematic dependence of the SXR peak flux - HXR fluence relationship on the flare intensity.

The relation between the SXR peak flux, $F_{\mathrm{P}, \mathrm{SXR}}$, and the maximum thermal energy, $\epsilon_{\mathrm{th} \text {,max }}$, approximated by the thermal energy at the time of the peak soft X-ray emission, can be expressed as (cf. Lee et al. 1995):

$\epsilon_{\mathrm{th}, \max } \propto \frac{F_{\mathrm{P}, \mathrm{SXR}} T_{\mathrm{P}}^{3 / 2}}{n_{\mathrm{P}} I\left(T_{\mathrm{P}}\right)}$,

where

$I(T)=\int_{K_{1} / k_{\mathrm{B}} T}^{K_{2} / k_{\mathrm{B}} T} G(\xi, T) \mathrm{e}^{-\xi} \mathrm{d} \xi$.

$T$ is the temperature, $n$ is the density, $k_{\mathrm{B}}$ is Boltzmann's constant, and $K_{1}$ and $K_{2}$ are the energy limits of the soft X-ray detector. The subscript "P" indicates the value of the respective quantities at the time of the peak SXR flux. Here $G$ denotes the ratio of the actual emission at energy $K$ to the emission that would be appropriate for classical thermal bremsstrahlung (for further discussion see Lee et al. 1995). The relationship between the HXR fluence, $\mathcal{F}_{\mathrm{HXR}}$, and the total energy deposited by nonthermal electrons, $\epsilon_{\mathrm{e}^{-}}$, is given by (cf. Lee et al. 1995):

$\epsilon_{\mathrm{e}^{-}} \propto \mathcal{F}_{\mathrm{HXR}}\left\langle\gamma^{2}(\gamma-1)\left(\frac{E_{0}}{K_{0}}\right)^{1-\gamma}\right\rangle$,

with $E_{0}$ the low energy cut-off of the accelerated electron spectrum, $K_{0}$ the photon energy, and $\gamma$ the photon spectral index. The angle brackets denote the flux-averaging, defined for a quantity $x(t)$ as:

$\langle x\rangle=\frac{\int_{t_{0}}^{t_{0}+D} x(t) F_{\mathrm{HXR}}(t) \mathrm{d} t}{\int_{t_{0}}^{t_{0}+D} F_{\mathrm{HXR}}(t) \mathrm{d} t}$.

If the Neupert effect for the energies as expected from the electron-beam-driven evaporation model obeys Eq. (3), then from Eqs. (6) and (8) it follows that the factor $k$, which relates the SXR peak flux and the HXR fluence (Eq. (1)), is given by:

$k \propto \frac{n_{\mathrm{P}} I\left(T_{\mathrm{P}}\right)}{T_{\mathrm{P}}^{3 / 2}}\left\langle\gamma^{2}(\gamma-1)\left(\frac{E_{0}}{K_{0}}\right)^{1-\gamma}\right\rangle$.
If the chromospheric evaporation model is valid for the considered flare sample, $k$ gives the productivity of SXR emission relative to the HXR emission. If any of the quantities on the right hand side of Eq. (10) depends systematically on the flare intensity, that must also be reflected in the behavior of $k$.

Feldman et al. (1996) found that the temperature of the SXR emitting plasma shows a tendency to increase with flare intensity. This would imply that $k$ should decrease with the importance of the SXR burst. However, Garcia (2000) reported recently that, not only the temperature but also the density of the SXR emitting plasma tends to increase with increasing flare intensity. So, in the first term on the right hand side of Eq. (10) the two effects could, at least partly, compensate for each other. Such an assumption is consistent with Fig. 9 (top panel), which shows that there is only a weak anti-correlation between $k$ and the SXR peak flux (see also Table 2, set 1).

All of this suggests that the second term on the right hand side of Eq. (10), which is representative for the HXR emission, might be responsible for a systematic dependence of $k$ on the flare intensity. This is also supported by the high anticorrelations between $k$ and the HXR fluence as well as $k$ and the HXR peak flux, in contrast to the poor anti-correlation between $k$ and the SXR peak flux (cf. Figs. 7-9, top panels; Table 2, set 1 ).

Little is known about the cut-off energy $E_{0}$, which in principle may vary from flare to flare (e.g., Wu et al. 1986). For the photon spectral index, $\gamma$, the situation is also rather unclear. Datlowe et al. (1974), Dennis (1985) and Bromund et al. (1995) did not find a correlation between the photon spectral index and the flare intensity. However, from statistical considerations Lee et al. (1995) inferred that a weak correlation between $\gamma$ and the HXR fluence might exist. Tomczak (1999), who studied selected flares observed by the Hard X-ray Telescope and the Soft X-ray Telescope aboard Yohkoh, found that smaller values of $\gamma$ (harder HXR energy spectrum) tend to be associated with higher hard X-ray intensities. Moreover, in this study it is reported that the relative productivity of soft X-rays with regard to hard X-rays, depends on the energy spectrum of the hard $\mathrm{X}$-ray photons. A steeper energy spectrum, i.e. larger $\gamma$, causes a higher soft X-ray productivity with respect to the hard X-ray. In principle, the results of Tomczak (1999) coincide with the findings of the present study that the relative productivity of SXR emission per HXR emission, i.e. $k$, is larger for weak HXR flares than for intense ones (see Figs. 7 and 8, top panels). However, it has to be stressed that the study of Tomczak (1999) is based only on a sample of five events. Moreover, in this paper the hard X-rays are primarily compared with SXR footpoint emission, which is only a small fraction of the total SXR emission. Thus, this study is only of limited relevance for the comparison of spatially integrated soft and hard X-rays, as performed in the present paper.

On the basis of frequency distributions of HXR fluences and SXR peak fluxes, Lee et al. (1995) derived a scaling of the SXR parameters, i.e. $n_{\mathrm{P}}, T_{\mathrm{P}}$ and $I\left(T_{\mathrm{P}}\right)$, inferring the HXR fluence - SXR peak flux relationship from the differences of the power-law indices describing the SXR and HXR frequency distributions. For the derivation of this scaling, two assumptions were made: a) The electron-beam-driven evaporation model, 
i.e. Eq. (3), holds for the bulk of flares; b) The parameters that determine the relation between the total energy deposited by electrons and the HXR emission, $\gamma$ and $E_{0}$, do not systematically depend on the flare intensity, i.e. $\epsilon_{\mathrm{e}^{-}} \propto \mathcal{F}_{\mathrm{HXR}}$, but only the parameters that interrelate the thermal energy and the SXR emission may vary with the flare intensity. As shown by the authors, the derived scaling does not agree with observations.

The present study suggests that this discrepancy probably arises due to inappropriate assumptions. We inferred that $\sim 25 \%$ of the events show strong deviations from the expectations of the electron-beam-driven evaporation model. So the first assumption by Lee et al. (1995) that the electron-beam-driven evaporation model statistically holds for all flares is unlikely to be fulfilled. Moreover, Figs. 7-9 (top panels) provide hints that the relation between SXR emission and thermal energy does not systematically (or only weakly) depend on the flare importance but the relation HXR emission - nonthermal energy does, just the opposite of the second assumption made by Lee et al. (1995).

Finally, let us note that flares are constituted of multitemperature plasma. McTiernan et al. (1999) have shown that the high-temperature component is more likely to exhibit the Neupert effect than the low-temperature component. In combination with the findings of Feldman et al. (1996) and Garcia (2000) that the temperature increases with the flare importance, this might imply that more intense flares should show a more pronounced Neupert effect. Indeed, Fig. 2 shows that for intense flares the HXR fluence - SXR peak flux relationship approaches a linear function.

\subsection{Interpretation in terms of an additional agent}

As mentioned in Sect. 5.3, the deviation from $k=$ const. can be also a result of an additional energy transport mechanism from the energy release site. Since the energy release site is strongly heated (see, e.g., Tsuneta 1996), a quite promising candidate for such energy transport mechanism is thermal conduction (for discussion see Vršnak 1989; Somov 1992; McDonald et al. 1999; and references therein), which is supported by various observations. From high time resolution HXR and H $\alpha$ observations, Kämpfer \& Magun (1983) found evidence for the occurrence of energy transport by electronbeams at one flare kernel and for conductive energy transport at another kernel. Moreover, various observations are indicative of prolonged chromospheric evaporation driven by thermal conduction fronts during the decaying phase of flares (e.g., Zarro \& Lemen 1988; Czaykowska et al. 2001). Recently, extensive simulations of chromospheric evaporation driven by thermal conduction have been performed by Yokoyama \& Shibata (1998, 2001).

Under such an assumption of an additional heating agent, the events of set 2 indicate that in a significant fraction of flares this additional heat input can be even more important than that associated with electron beams. The other extreme is where electron-beam-driven evaporation is the dominant energy transport mechanism, i.e. the second-agent contribution is negligible. However, these flares are probably not the only

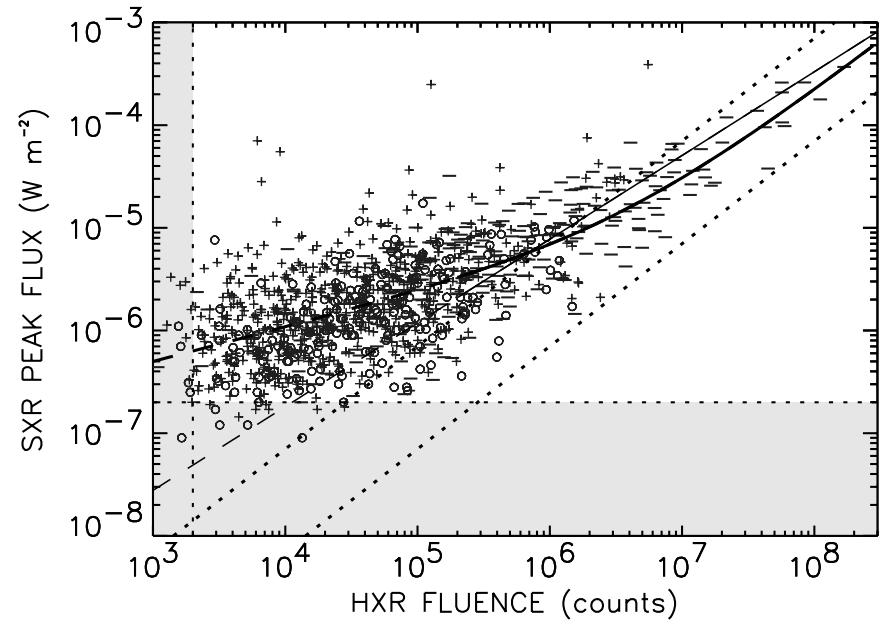

Fig. 11. Scatter plot of the SXR peak flux versus the HXR fluence for the whole sample. Several curves are overplotted: the functional fit according to Eq. (11), represented by the curved line, and a linear fit (thin solid line), both derived for $\mathcal{F}_{\mathrm{HXR}}>2 \times 10^{5}$ counts, the extension of the fits to smaller fluences is indicated by dashed lines; two lines of constant $k$, with $k=7 \times 10^{-12}$ (upper dotted line) and $k=0.7 \times 10^{-12}$ (lower dotted line).

constituents of set 1 , but it may embrace also flares, in which the energy deposition by high energy electrons and those by the second agent have roughly the same time evolution.

To some degree, it is also expected that a Neupert-like relationship between the hard and soft X-ray emissions shows up in thermal conduction models (Dennis \& Schwartz 1989). For example, it can be expected that a more powerful energy release results in a higher temperature in the primary energy release site and thus a higher thermal conduction flux. On the other hand, a powerful energy release also implies higher electric fields in the primary energy release site and thus a more efficient electron acceleration. Under such circumstances, it can be assumed that the relationship between $F_{\mathrm{P}, \mathrm{SXR}}$ and $\mathcal{F}_{\mathrm{HXR}}$ has the form:

$F_{\mathrm{P}, \mathrm{SXR}}=\tilde{k} \cdot \mathcal{F}_{\mathrm{HXR}}+\tilde{k}^{*} \cdot\left(\mathcal{F}_{\mathrm{HXR}}\right)^{\delta}$.

The first term on the right hand side represents the electronbeam-driven contribution. The second term describes the contribution by thermal conduction, which may (presumably weakly) depend on the HXR flare importance. Here it is assumed that the coefficients $\tilde{k}$ and $\tilde{k}^{*}$ may differ from flare to flare depending on physical conditions, but do not systematically vary with the flare intensity. Under such an additional agent assumption, set 1 and set 2 represent just the extremes of a continuum regarding the two different types of energy input driving the evaporation, i.e. electron beams and thermal conduction, and the whole sample of events should be considered together.

We applied a least-squares fit in log-log space with the functional form given in Eq. (11), whereas only events with $\mathcal{F}_{\text {HXR }}>2 \times 10^{5}$ counts were taken into account, as for this range any influence due to observational thresholds can be excluded (cf. Fig. 2). From the fit we obtained: $\tilde{k}=1.68 \times 10^{-12}$, $\tilde{k}^{*}=1.27 \times 10^{-8}$ and $\delta=0.45$. We note that, qualitatively 
speaking, the fit yields a similar result if it is applied to the events of set 1 only $\left(\tilde{k}=1.49 \times 10^{-12}, \tilde{k}^{*}=1.59 \times 10^{-8}\right.$, $\delta=0.44)$. For comparison, we have also applied a linear fit in log-log space, obtaining a slope $b=0.83 \pm 0.03$ in the range $\mathcal{F}_{\text {HXR }}>2 \times 10^{5}$ counts. It is worth mentioning that the linear fit for events with $\mathcal{F}_{\text {HXR }}>3 \times 10^{6}$ counts gives a slope of almost $1(b=0.96 \pm 0.07)$, consistent with a linear interpretation of the Neupert effect for these most intense events. Figure 11 shows the scatter plot of the SXR peak flux versus the HXR fluence for the complete sample (note that the data are identical to Fig. 2) in combination with the functional fit of Eq. (11) as well as the linear fit, derived from the events with $\mathcal{F}_{\text {HXR }} \gtrsim 2 \times 10^{5}$ counts. The range, from which the fit was derived, is drawn in solid lines; the extension of the fit to smaller fluences is represented by dashed lines.

As it can be seen from Fig. 11, Eq. (11) approaches a slope of 1 at large fluences. For small fluences, the fit probably yields too high SXR peak fluxes, assuming that a considerable part of flares lies below the indicated SXR threshold. According to the interpretation of Eq. (11) as a sum of two different energy contributions to the evaporation, i.e. electron beams and thermal conduction, the derived fit values imply that the electronbeam component is only dominating for flares with $\mathcal{F}_{\mathrm{HXR}} \gtrsim$ $1.0 \times 10^{7}$ counts or, accordingly, $F_{\text {SXR,P }} \gtrsim 1.4 \times 10^{-5} \mathrm{~W} \mathrm{~m}^{-2}$. These are rather improbably high values, as they imply that the electron-beam-driven component is only dominating for the most intense events. However, we stress that it is somewhat risky to apply such a functional fit to a limited data set, and the derived fit values have to be taken with caution. On the other hand, it has to be noted that the increasing slope indicated by this functional fit is also reflected by the linear fit over different fluence ranges. As shown above, for very large fluences ( $\mathcal{F}_{\text {HXR }} \gtrsim 3 \times 10^{6}$ counts), the slope is almost 1 , whereas it is significantly lower if less intense flares are also taken into account.

In Fig. 11 we have also drawn a line of constant $k$, with $k=0.7 \times 10^{-12}$. As can be seen from the figure, this value represents a lower limit of $k$. On the one hand, the line gives an impression, in which range we might have missed events due to observational selection effects. Furthermore, and more importantly, the existence of such a lower limit for $k$ (which is quite sharply defined), contains very physical information: electron beams always produce at a least a certain amount of soft X-rays (see the "white" triangle below the line $k=0.7 \times 10^{-12}$ ). There are no events with large HXR fluences but low soft X-ray emission. On the other hand, the upper limit of $k$ is less well defined. There are events with small HXR fluences but comparatively large SXR peak fluxes, indicative for an additional agent besides the HXR emitting electrons contributing to the energy of the SXR emitting plasma. Moreover, the upper limit of $k$ cannot be approximated by a line of constant $k$ (except, maybe, for flares with very large fluences), but it reveals an increasing slope with increasing HXR fluence. A similar shape can be detected in Fig. 5.4.2 from Wu et al. (1986), who have drawn an analogous scatter plot for a selected sample of 101 events measured by the HXRBS and the BCS instrument aboard the Solar Maximum Mission. It has to be noted that the shape of this upper limit cannot be influenced by sensitivity effects, but it reflects a real physical phenomenon, i.e. a different behavior of the SXR peak flux - HXR fluence relationship for small and large flares.

We have drawn also a line of constant $k$ in Fig. 11 with $k=$ $7 \times 10^{-12}$, which represents an estimate of the upper limit of $k$ for events with $\mathcal{F}_{\mathrm{HXR}} \gtrsim 10^{7}$ counts. Thus, for the largest events, the range between the lower and the upper limit of $k$ covers about one order of magnitude. Approaching smaller fluences, there is an increasing number of flares lying above this range, i.e. at higher SXR fluxes, violating the linear Neupert relation.

\section{Conclusions}

In the following we briefly summarize the basic results of the analysis:

1. The distribution of the differences of the SXR peak times and HXR end times is strongly peaked at $\Delta t=0$.

2. Yet, a significant fraction of events $(\sim 25 \%)$ shows strong deviations from the chosen timing criterion applied as a necessary condition for consistency with the Neupert effect (set 2). These events are characterized by increasing SXR emission beyond the end of the HXR emission.

3. Flares that satisfy the timing criterion (set 1), embracing about one half of the events, reveal a much higher correlation between the HXR fluence and the SXR peak flux than those of set 2 . The strong correlations found for set 1 suggests that electron-beam-driven chromospheric evaporation plays an important role for these events.

4. Set 2 contains many fewer large events than set 1 . For weak flares, on average, the events of set 2 have higher SXR peak fluxes at a given HXR fluence than those of set 1, suggesting that an additional energy transport mechanism other than the HXR emitting electrons contributes to the SXR emission.

5. Events with negative $\Delta t$, i.e. the SXR peak occurs before the end of the HXR emission, preferentially belong to set 1 and are of long duration. These events are compatible with the electron-beam-driven evaporation model. In the decay phase of long-duration flares, the instantaneous cooling of the hot plasma is likely to dominate over the evaporationdriven energy supply (Li et al. 1993). From the present data set we infer that, on average, this phase covers $\sim 0.4$ times of the HXR event duration.

6. For the events of set 1 , the SXR peak flux - HXR fluence relationship is not linear. However, for large HXR fluences the SXR peak flux - HXR fluence relationship tends towards a linear function. Correspondingly, for the events of set 1 , the factor $k$ is a decreasing function of the HXR fluence. Yet, for large HXR fluences, $k$ becomes approximately constant.

7. Although high correlations are found among the SXR peak fluxes and HXR fluences, the scatter in the SXR peak flux versus HXR fluence plot is larger than an order of magnitude (up to two orders of magnitude).

Finally, we stress that although the results presented show that in a statistical sense about half of the events show characteristics compatible with the Neupert effect, the scatter of the SXR 
peak flux versus HXR fluence indicates that a wide range of physical conditions are met in solar flares. The main outcomes of the analysis can be interpreted in the sense that the process of electron-beam-driven evaporation plays an important role in solar flares. On the other hand, the prolonged SXR emission found in a significant fraction of events also gives strong indications for the presence of an additional energy transport mechanism, probably thermal conduction, whereas the relative contribution of the different transport mechanisms shows a dependence on the flare importance. The energy provided by the additional agent may play a prominent role in weak flares, whereas in intense events its contribution is much less important than the electron-beam-driven component.

Acknowledgements. The authors thank Helen Coffey from NGDC for making available the SXR data, and the BATSE team for the access to the HXR data. We also thank the referee Jim McTiernan for thoughtful comments. A. V., M. T. and A. H. gratefully acknowledge the Austrian Fonds zur Förderung der wissenschaftlichen Forschung (FWF grants P13653-PHY and P15344-PHY) for supporting this project. B. V. acknowledges the University of Graz for financial support and is grateful to the colleagues from the Institute for Geophysics, Astrophysics and Meteorology for their hospitality.

\section{References}

Antonucci, E., Gabriel, A. H., \& Dennis, B. R. 1984, ApJ, 287, 917

Antonucci, E., Alexander, D., Culhane, J. L., et al. 1999, in The Many Faces of the Sun: A Summary of the Results from NASA's Solar Maximum Mission, ed. K. T. Strong, J. L. R. Saba, B. M. Haisch, \& J. T. Schmelz (Springer-Verlag), 331

Bromund, K. R., McTiernan, J. M., \& Kane, S. R. 1995, ApJ, 455, 733

Brown, J. C. 1971, Solar Phys., 18, 489

Crosby, N. B., Vilmer, N., Lund, N., \& Sunyaev, R. 1998, A\&A, 334, 299

Czaykowska, A., Alexander, D., \& De Pontieu, B. 2001, ApJ, 552, 849

Datlowe, D. W., Hudson, H. S., \& Peterson, L. E. 1974, Solar Phys., 35,193

Dennis, B. R. 1985, Solar Phys., 100, 465

Dennis, B. R. 1988, Solar Phys., 118, 49

Dennis, B. R., \& Schwartz, R. A. 1989, Solar Phys., 121, 75

Dennis, B. R., Uberall, B. M., \& Zarro, D. M. 1992, in Eruptive Solar Flares, Proc. IAU Coll. 133, ed. Z. Svestka, B. V. Jackson, \& M. E. Machado (Springer-Verlag, New York), 139

Dennis, B. R., \& Zarro, D. M. 1993, Solar Phys., 146, 177

Donelly, R. F., \& Unzicker, A. 1974, NOAA Tech. Memo ELR SEL72

Feldman, U., Doschek, G. A., Behring, W. E., \& Phillips, K. J. H. 1996, ApJ, 460, 1034
Fisher, G. H., Canfield, R. C., \& McClymont, A. N. 1985, ApJ, 289, 425

Fishman, G. J., Meegan, C. A., Wilson, R. B., et al. 1989, in Max '91 Workshop 2: Developments in Observations and Theory for Solar Cycle 22, 96

Fishman, G. J., Meegan, C. A., Wilson, R. B., Paciesas, W. S., \& Pendleton, G. N. 1992, in The Compton Observatory Science Workshop, NASA CP 3137, 26

Gan, W.-Q., Li, Y.-P., Chang, J. 2002, Chin. J. Astron. \& Astrophys., 2,103

Garcia, H. A. 1994, Solar Phys., 154, 275

Garcia, H. A. 2000, ApJS, 127, 189

Güdel, M., Benz, A. O., Schmitt, J. H. M. M., \& Skinner, S. L. 1996, ApJ, 471, 1002

Hawley, S. L., Fisher, G. H., Simon, T., et al. 1995, ApJ, 453, 464

Isobe, K., Feigelson, E. D., Akritas, M. G., \& Babu, G. J. 1990, ApJ, 364, 104

Kämpfer, N., \& Magun, A. 1983, ApJ, 274, 910

Lee, T. T., Petrosian, V., \& McTiernan, J. M. 1993, ApJ, 412, 401

Lee, T. T., Petrosian, V., \& McTiernan, J. M. 1995, ApJ, 448, 915

Li, P., Emslie, A. G., \& Mariska, J. T. 1993, ApJ, 417, 313

McDonald, L., Harra-Murnion, L. K., \& Culhane, J. L. 1999, Solar Phys., 185, 323

McTiernan, J. M. 1999, AAS, 194.5402M

McTiernan, J. M., Fisher, G. H., \& Li, P. 1999, ApJ, 514, 472

Neupert, W. M. 1968, ApJ, 153, L59

Petrosian, V. 1994, in ed. S. Enome, \& T. Hirayama, in Proc. Kofu Symposium, Nobeyama Radio Obs. Rep., No. 360, 239

Plunkett, S. P., \& Simnett, G. M. 1994, Solar Phys., 155, 351

Schwartz, R. A., Dennis, B. R., Fishman, G. J., et al. 1992, in The Compton Observatory Science Workshop, NASA CP 3137, 457

Simnett, G. M. 1986, Solar Phys., 106, 165

Somov, B. V. 1992, Physical Processes in Solar Flares, Astrophysics and Space Science Library, vol. 172 (Kluwer Academic Publishers)

Starr, R., Heindl, W. A., Crannel, C. J., et al. 1988, ApJ, 329, 967

Tanaka, K., Nitta, N., \& Watanabe, T. 1982, in Hinotori Symposium on Solar Flares, 20

Tomczak, M. 1999, A\&A, 342, 583

Tsuneta, S. 1996, ApJ, 456, 840

Veronig, A., Temmer, M., Hanslmeier, A., Otruba, W., \& Messerotti, M. 2002a, A\&A, 382, 1070

Veronig, A., Vršnak, B., Temmer, M., \& Hanslmeier, A. 2002b, Solar Phys., in press

Vršnak, B. 1989, Solar Phys., 120, 79

Wu, S. T., de Jager, C., Dennis, B. R., et al. 1986, in Energetic Phenomena on the Sun, Astrophysics and Space Science Library, vol. 153, ed. M. R. Kundu, \& B. E. Woodgate (Kluwer Academic Publishers), 383

Yokoyama, T., \& Shibata, K. 1998, ApJ, 494, L113

Yokoyama, T., \& Shibata, K. 2001, ApJ, 549, 1160

Zarro, D., \& Lemen, J. 1988, ApJ, 329, 456 